\title{
Research Article \\ Effect of Rotation and Magnetic Field through Porous Medium on Peristaltic Transport of a Jeffrey Fluid in Tube
}

\author{
S. R. Mahmoud ${ }^{1,2}$ \\ ${ }^{1}$ Mathematics Department, Faculty of Science, King Abdulaziz University, Jeddah 21589, Saudi Arabia \\ ${ }^{2}$ Mathematics Department, Faculty of Science, Sohag University, Sohag 82524, Egypt
}

Correspondence should be addressed to S. R. Mahmoud, srhassan@kau.edu.sa

Received 8 June 2011; Accepted 25 July 2011

Academic Editor: Angelo Luongo

Copyright (c) 2011 S. R. Mahmoud. This is an open access article distributed under the Creative Commons Attribution License, which permits unrestricted use, distribution, and reproduction in any medium, provided the original work is properly cited.

This paper is concerned with the analysis of peristaltic motion of a Jeffrey fluid in a tube with sinusoidal wave travelling down its wall. The effect of rotation, porous medium, and magnetic field on peristaltic transport of a Jeffrey fluid in tube is studied. The fluid is electrically conducting in the presence of rotation and a uniform magnetic field. An analytic solution is carried out for long wavelength, axial pressure gradient, and low Reynolds number considerations. The results for pressure rise and frictional force per wavelength were obtained, evaluated numerically, and discussed briefly.

\section{Introduction}

The dynamics of the fluid transport by peristaltic motion of the confining walls has received a careful study in the literature. The need for peristaltic pumping may arise in circumstances where it is desirable to avoid using any internal moving parts such as pistons in a pumping process. The peristalsis is also well known to the physiologists to be one of the major mechanisms of fluid transport in a biological system and appears in urine transport from kidney to bladder through the ureter, movement of chyme in the gastrointestinal tract, the movement of spermatozoa in the ductus efferentes of the male reproductive tract and the ovum in the female fallopian tube, the locomotion of some worms, transport of lymph in the lymphatic vessels, and vasomotion of small blood vessels such as arterioles, venules, and capillaries. Technical roller and finger pumps also operate according to this rule. The behavior of most of the physiological fluids is known to be non-Newtonian. Several models have been proposed to explain the non-Newtonian behavior of fluids. 
Mahmoud et al. [1-3] investigated effect of the rotation on the radial vibrations in a nonhomogeneous orthotropic hollow cylinder and effect of the rotation on wave motion through cylindrical bore in a micropolar porous cubic crystal, and he investigated effect of the rotation on the radial vibrations in a nonhomogeneous orthotropic hollow cylinder. Abd-Alla et al. [4-7] investigated effect of the rotation on a nonhomogeneous infinite cylinder of orthotropic material, influences of rotation, magnetic field, initial stress and gravity on rayleigh waves in a homogeneous orthotropic elastic half space, and magneto-thermoelastic problem in rotating nonhomogeneous orthotropic hollow cylindrical under the hyperbolic heat conduction model, and they studied effect of the rotation on propagation of thermoelastic waves in a nonhomogeneous infinite cylinder of isotropic material. Mahmoud [8] studied effect of rotation on generalized magneto-thermoelastic Rayleigh waves in a granular medium under influence of gravity field and initial stress. Afifi et al. [9-11] investigated effect of magnetic field and wall properties on peristaltic motion of micropolar fluid in circular cylindrical tubes and interaction of peristaltic flow with pulsatile magnetofluid through a porous medium, and they studied aspects of a magnetofluid with suspended particles. Various attempts [12-14] are made to solve the extremely complex equations of motion of non-Newtonian fluids. The good number of recent investigations [15-23] on the peristalsis of non-Newtonian fluids has been presented with various perspectives, in channels or tubes. Most of the analytic studies are asymptotic expansions with small Reynolds number, wave number, and amplitude ratio as a perturbation parameter. Siddiqui et al. [24] examined the peristaltic motion of a magnetohydrodynamic Newtonian fluid in a tube by taking long wavelength approximation. More recently Hayat and Ali [22] studied the peristaltic motion of a third-order fluid in a tube under long wavelength and small Deborah number approximation. However, no attempt has been made to discuss the peristaltic motion of a magnetohydrodynamic (MHD) non-Newtonian fluid in a tube which holds for all values of non-Newtonian parameters. In the present analysis, such an attempt has been made. The liquid considered is of the Jeffrey type and is electrically conducting. This shows worthwhile the first attempt for MHD non-Newtonian flow in a tube for all values of the rheological parameters. The Jeffrey model is relatively simpler linear model using time derivatives instead of convected derivatives, for example, what the Oldroyd-B model does; it represents a rheology different from the Newtonian. Although more sophisticated viscoelastic models than the Jeffrey model exist, in a first study of the MHD peristaltic motion of a non-Newtonian fluid in circular cylindrical tube, the choice of Jeffrey fluid model is motivated by the following.

In spite of its relative simplicity, the Jeffrey model can indicate the changes of the rheology on the peristaltic flow even under the assumption of large wavelength, low Reynolds number, and small or large amplitude ratio. In Newtonian fluid, Mekheimer [25] studied the MHD peristaltic flow in a channel under the assumption of small wave number. Therefore, at least in an initial study, this motivates an analytic study of MHD peristaltic nonNewtonian tube flow that holds for all non-Newtonian parameters. By choosing the Jeffrey fluid modele it became possible to treat both the MHD Newtonian and non-Newtonian problems analytically under long wavelength and low Reynolds number consideration. Considering the blood as an MHD fluid, it may be possible to control blood pressure and its flow behavior by using an appropriate magnetic field. The influence of magnetic field may also be utilized as a blood pump for cardiac operations for blood flow in arterial stenosis or arteriosclerosis. 


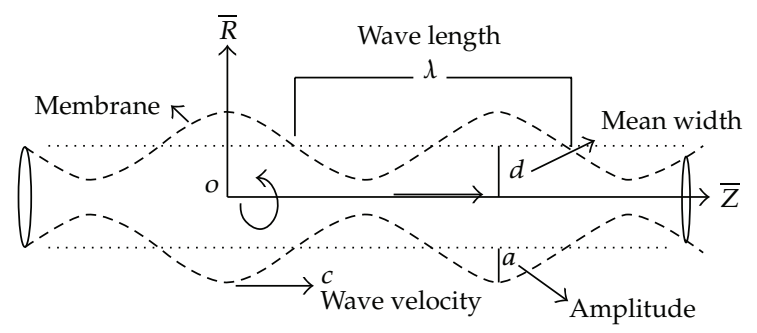

Figure 1: Geometry of peristaltic motion on asymmetric channel through porous medium.

\section{Formulation of the Problem}

Consider the axisymmetric flow of a Jeffrey fluid in a uniform circular tube with a sinusoidal peristaltic wave of small amplitude travelling down its wall (see Figure 1). The geometry of wall surface is therefore described as

$$
\bar{h}(\bar{z}, \bar{t})=d+a \cos \left[\frac{2 \pi}{\lambda}(\bar{z}-c \bar{t})\right] .
$$

Here $a$ is amplitudes of the waves, $\lambda$ is the wavelength, $d$ is average radius of the undisturbed tube. The constitutive equations for an incompressible Jeffrey fluid are:

$$
\begin{gathered}
\bar{I}_{0}=-\bar{p} \bar{I}+\bar{S}, \\
\bar{S}=\frac{\mu}{1+\lambda_{1}}\left(\overline{\dot{\gamma}}+\lambda_{2} \overline{\ddot{\gamma}}\right),
\end{gathered}
$$

where $\bar{I}_{0}$ and $\bar{S}$ are Cauchy stress tensor and extra stress tensor, respectively, $\bar{p}$ is the pressure, $\bar{I}$ is the identity tensor, $\mu$ is dynamic viscosity, $\lambda_{1}$ is the ratio of relaxation to retardation times, $\lambda_{2}$ is the retardation time, $\overline{\dot{\gamma}}$ is the shear rate, and dots over the quantities indicate differentiation with respect to time. In laboratory frame, the equations governing twodimensional motion of an incompressible MHD Jeffrey fluid through a porous medium [24] are as follows:

$$
\begin{gathered}
\frac{\partial \bar{U}}{\partial \bar{R}}+\frac{\partial \bar{W}}{\partial \bar{Z}}+\frac{\bar{U}}{\bar{R}}=0 \\
\rho \frac{\partial \bar{U}}{\partial \bar{t}}+\bar{U} \frac{\partial \bar{U}}{\partial \bar{R}}+\bar{W} \frac{\partial \bar{U}}{\partial \bar{Z}}+\bar{\Omega}^{2} \bar{U}=-\frac{\partial \bar{p}}{\partial \bar{R}}+\frac{1}{\bar{R}} \frac{\partial}{\partial \bar{R}}\left(\bar{R} \frac{\partial \bar{S}_{\bar{R} \bar{R}}}{\partial \bar{R}}\right)-\frac{\bar{S}_{\bar{R} \bar{R}}}{\bar{R}^{2}}+\frac{\partial^{2} \bar{S}_{\bar{R} \bar{Z}}}{\partial \bar{Z}^{2}}-\frac{\mu}{\bar{\kappa}_{0}} \bar{U} \\
\rho\left(\frac{\partial \bar{W}}{\partial \bar{t}}+\bar{U} \frac{\partial \bar{W}}{\partial \bar{R}}+\bar{W} \frac{\partial \bar{W}}{\partial \bar{Z}}\right)=-\frac{\partial \bar{p}}{\partial \bar{Z}}+\frac{1}{\bar{R}} \frac{\partial}{\partial \bar{R}}\left(\bar{R} \frac{\partial \bar{S}_{\bar{R} \bar{Z}}}{\partial \bar{R}}\right)+\frac{\partial^{2} \bar{S}_{\bar{Z} \bar{Z}}}{\partial \bar{Z}^{2}}-\sigma B_{0}^{2} \bar{W}-\frac{\mu}{k_{0}} \bar{W}+\rho \bar{\Omega}^{2} \bar{W}
\end{gathered}
$$

where $\bar{R}, \bar{W}$ are the velocity components in the laboratory frame $(\bar{R}, \bar{Z}), \rho$ is the density, $\bar{p}$ is the pressure, $\sigma$ is the electrical conductivity of the fluid, $B_{0}$ is a constant of magnetic field, $\mu$ is 
the kinematic viscosity, $\Omega$ is the rotation component, and $k_{0}$ is the permeability of the porous medium, and we get [25]

$$
\begin{gathered}
\bar{S}=\frac{\mu}{1+\lambda_{1}}\left(1+\lambda_{2}\left[\bar{U} \frac{\partial}{\partial \bar{R}}+\bar{W} \frac{\partial}{\partial \bar{Z}}\right]\right) \overline{\dot{\gamma}} \\
\bar{S}_{\bar{R} \bar{R}}=\frac{2 \mu}{1+\lambda_{1}}\left(1+\lambda_{2}\left[\bar{U} \frac{\partial}{\partial \bar{R}}+\bar{W} \frac{\partial}{\partial \bar{Z}}\right]\right) \frac{\partial \bar{U}}{\partial \bar{R}} \\
\bar{S}_{\bar{Z} \bar{Z}}=\frac{2 \mu}{1+\lambda_{1}}\left(1+\lambda_{2}\left[\bar{U} \frac{\partial}{\partial \bar{R}}+\bar{W} \frac{\partial}{\partial \bar{Z}}\right]\right) \frac{\partial \bar{U}}{\partial \bar{Z}} \\
\bar{S}_{\bar{R} \bar{Z}}=\frac{\mu}{1+\lambda_{1}}\left(1+\lambda_{2}\left[\bar{U} \frac{\partial}{\partial \bar{R}}+\bar{W} \frac{\partial}{\partial \bar{Z}}\right]\right)\left(\frac{\partial \bar{U}}{\partial \bar{Z}}-\frac{\partial \bar{W}}{\partial \bar{R}}\right) .
\end{gathered}
$$

We will carry out this investigation in a coordinate system moving with the wave speed in which the boundary shape is stationary. The coordinates and velocities in the laboratory frame $(\bar{R}, \bar{Z})$ and the wave frame $(\bar{x}, \bar{y})$, are related by

$$
\bar{r}=\bar{R}-c \bar{t}, \quad \bar{z}=\bar{Z}, \quad \bar{u}=\bar{U}-c, \quad \bar{w}=\bar{W}, \quad \bar{p}=\bar{p}(\bar{R}, t),
$$

where $\bar{u}, \bar{w}$ are the velocity components in the wave frame $(\bar{r}, \bar{z})$. We introduce the following nondimensional variables and parameters for the flow:

$$
\begin{gathered}
r=\frac{\bar{R}}{d_{1}}, \quad z=\frac{2 \pi \bar{Z}}{\lambda}, \quad u=\frac{\bar{U}}{c \delta}, \quad w=\frac{\bar{W}}{c}, \quad \delta=\frac{2 \pi d_{1}}{\lambda}, \quad t=\frac{2 \pi c \bar{t}}{\lambda}, \\
p=\frac{2 \pi d_{1}^{2} \bar{p}}{\mu c \lambda}, \quad H=\frac{\sigma}{\mu} B_{0}^{2} d_{1}^{2}, \quad \operatorname{Re}=\frac{\rho c d_{1}}{\mu}, \quad S=\frac{d_{1}}{\mu c}, \\
\Omega^{2}=\frac{\bar{\Omega}^{2}}{v} d_{1}^{2}, \quad \bar{k}=\frac{k_{0}}{d_{1}^{2}}, \quad h_{1}=\frac{\bar{h}_{1}}{d_{1}}, \quad a=\frac{a_{1}}{d_{1}},
\end{gathered}
$$

where Re is the Reynolds number, $\delta$ is the dimensionless wave number, and $H$ is the magnetic parameter (Hartman number). Using nondimensional variables and parameters in (2.3), we get the following:

$$
\begin{gathered}
\delta \frac{\partial u}{\partial r}+\delta \frac{\partial W}{\partial Z}+\delta \frac{u}{r}=0 \\
\operatorname{Re} \delta\left(u \frac{\partial}{\partial r}+w \frac{\partial}{\partial z}\right) u=-\frac{\partial p}{\partial r}+\delta^{2}\left[\frac{1}{r} \frac{\partial}{\partial r}\left(r \frac{\partial S_{r r}}{\partial r}\right)-\frac{S_{r r}}{r^{2}}+\delta^{2} \frac{\partial^{2} S_{r z}}{\partial z^{2}}\right]-\frac{1}{\bar{k}} u-\Omega^{2} u \\
\operatorname{Re} \delta^{3}\left(u \frac{\partial}{\partial r}+w \frac{\partial}{\partial z}\right) w=-\frac{\partial p}{\partial z}+\frac{1}{r} \frac{\partial}{\partial r}\left(r \frac{\partial S_{r z}}{\partial r}\right)+\delta^{2} \frac{\partial^{2} S_{z z}}{\partial z^{2}}-H^{2} w-\frac{1}{\bar{k}} w+\Omega^{2} w
\end{gathered}
$$


introducing the stream function $\psi$ as

$$
u(r, z)=\frac{\delta}{r} \frac{\partial \psi}{\partial z}, \quad w(r, z)=-\frac{1}{r} \frac{\partial \psi}{\partial r}
$$

We can write (2.7) as follows:

$$
\begin{aligned}
\delta^{2} \frac{\partial}{\partial r}\left(\frac{1}{r} \frac{\partial \psi}{\partial z}\right)-\delta & \frac{\partial}{\partial z}\left(\frac{1}{r} \frac{\partial \psi}{\partial r}\right)+\delta^{2} \frac{\partial}{r^{2}}\left(\frac{1}{r} \frac{\partial \psi}{\partial z}\right)=0 \\
\operatorname{Re} \delta\left(\frac{\delta}{r} \frac{\partial \psi}{\partial z} \frac{\partial}{\partial r}-\frac{1}{r} \frac{\partial \psi}{\partial r} \frac{\partial}{\partial z}\right) \frac{1}{r} \frac{\partial \psi}{\partial z}= & -\frac{\partial p}{\partial r}+\delta^{2}\left[\frac{1}{r} \frac{\partial}{\partial r}\left(r \frac{\partial S_{r r}}{\partial r}\right)-\frac{S_{r r}}{r^{2}}+\delta^{2} \frac{\partial^{2} S_{r z}}{\partial z^{2}}\right] \\
& -\frac{\delta}{\bar{k}}\left(\frac{1}{r} \frac{\partial \psi}{\partial z}\right)-\delta \Omega^{2}\left(\frac{1}{r} \frac{\partial \psi}{\partial z}\right) \\
\operatorname{Re} \delta^{3}\left(\frac{\delta}{r} \frac{\partial \psi}{\partial z} \frac{\partial}{\partial r}+\frac{1}{r} \frac{\partial \psi}{\partial r} \frac{\partial}{\partial z}\right) \frac{1}{r} \frac{\partial \psi}{\partial r}= & -\frac{\partial p}{\partial z}+\frac{1}{r} \frac{\partial}{\partial r}\left(r \frac{\partial S_{r z}}{\partial r}\right)+\delta^{2} \frac{\partial^{2} S_{z z}}{\partial z^{2}} \\
& +H^{2}\left(\frac{1}{r} \frac{\partial \psi}{\partial r}\right)+\frac{1}{\bar{k}}\left(\frac{1}{r} \frac{\partial \psi}{\partial r}\right)-\Omega^{2}\left(\frac{1}{r} \frac{\partial \psi}{\partial r}\right) .
\end{aligned}
$$

Eliminating pressure from (2.9), (2.11) by cross-differentiation, using the long wavelength $(\delta \ll 1)$ and low Reynolds number in (2.9)-(2.11), and neglecting $\delta$ and higher power, we obtain

$$
\begin{gathered}
\frac{\partial p}{\partial r}=0 \\
\frac{\partial p}{\partial z}=\frac{1}{r} \frac{\partial}{\partial r}\left(\frac{r}{\left(1+\lambda_{1}\right)}\left(\frac{1}{r} \frac{\partial \psi}{\partial r}\right)\right)-\omega^{2}\left(\frac{1}{r} \frac{\partial \psi}{\partial r}\right),
\end{gathered}
$$

where $\omega^{2}=1 / \bar{k}-\Omega^{2}+H^{2}$,

$$
\begin{aligned}
& S_{r r}=\frac{2 \delta}{1+\lambda_{1}}\left[1+\frac{\delta c \lambda_{2}}{d_{1}}\left(\frac{\partial \psi}{\partial z} \frac{\partial}{\partial r}-\frac{\partial \psi}{\partial r} \frac{\partial}{\partial z}\right)\right] \frac{\partial}{\partial r}\left(\frac{1}{r} \frac{\partial \psi}{\partial z}\right) \\
& S_{z z}=\frac{2 \delta}{1+\lambda_{1}}\left[1+\frac{\delta c \lambda_{2}}{d_{1}}\left(\frac{\partial \psi}{\partial z} \frac{\partial}{\partial r}-\frac{\partial \psi}{\partial r} \frac{\partial}{\partial z}\right)\right] \frac{\partial}{\partial z}\left(\frac{1}{r} \frac{\partial \psi}{\partial r}\right)
\end{aligned}
$$

From (2.12) we show that $p \neq p(r)$. Differentiating (2.13) with respect to $r$ we get

$$
\frac{\partial}{\partial r}=\left(\frac{1}{r} \frac{\partial}{\partial r}\left(r \frac{\partial}{\partial r}\left(\frac{1}{r} \frac{\partial \psi}{\partial r}\right)\right)\right)-x^{2}\left(\frac{1}{r} \frac{\partial \psi}{\partial r}\right)=0
$$

where $x^{2}=\left(1+\lambda_{1}\right) \omega^{2}$. 


\section{Rate of Volume Flow}

The instantaneous volume flow rate in fixed coordinate system is given by

$$
\bar{Q}(\bar{z}, \bar{t})=2 \pi \int_{0}^{\bar{h}} \bar{w}(\bar{Z}, \bar{t}) \bar{R} d \bar{R}
$$

where $\bar{h}$ is a function of $\bar{Z}$ and $\bar{t}$. On substituting (2.5) into (3.1) and then integrating, one obtains

$$
\bar{Q}=\bar{q}+\pi c \bar{h}^{2}
$$

where

$$
\bar{q}=2 \pi \int_{0}^{\bar{h}} \bar{w} \bar{r} d \bar{r}
$$

is the volume flow rate in the moving coordinate system and is independent of time. Here, $\bar{h}$ is a function of $z$ alone. Using the dimensionless variables, we find

$$
F=\frac{\bar{q}}{2 \pi c a_{1}^{2}}=\int_{0}^{h} w r d r
$$

The time-mean flow over a period $T=\lambda / c$ at a fixed Z-position is defined as

$$
Q=\frac{1}{T} \int_{0}^{T} \bar{Q} d \bar{t}
$$

Using (3.2) into (3.5), $0<\epsilon<1$, we obtain

$$
Q=\bar{q}+\pi c a^{2}\left(1+\frac{\epsilon^{2}}{2}\right)
$$

Using dimensionless variables we write:

$$
\frac{Q}{2 \pi c a^{2}}=\frac{\bar{q}}{2 \pi c a^{2}}+\frac{1}{2}\left(1+\frac{\epsilon^{2}}{2}\right) .
$$

Equation (3.6) becomes

$$
\begin{gathered}
\beta=F+\frac{1}{2}\left(1+\frac{\epsilon^{2}}{2}\right), \\
\beta=\frac{Q}{2 \pi c \partial^{2}}, \quad F=\int_{0}^{h} \frac{\partial \psi}{\partial r} d r=\psi(h)-\psi(0),
\end{gathered}
$$

where $\beta$ and $F$ are, respectively, the flow rates in the fixed and wave frames. 
We note that $h$ represents the dimensionless form of the surface of the peristaltic wall:

$$
h(z)=1+\epsilon \cos 2 \pi z, \quad \epsilon=\frac{a_{1}}{d_{1}} .
$$

Choosing the zero value of the streamline along the central line $(w=0)$, we have $\psi(0)=0$. Then the shape of the wave at the boundary is the streamline with value $\psi(h)=F$ in wave frame, the boundary conditions in terms of stream

$$
\begin{gathered}
\psi=0, \quad \frac{\partial}{\partial r}\left(\frac{1}{r} \frac{\partial \psi}{\partial r}\right)=0, \quad \text { at } r=0, \\
\psi=F, \quad \frac{1}{r} \frac{\partial \psi}{\partial r}=1, \quad \text { at } r=h .
\end{gathered}
$$

\section{Method of Solution}

Integration of (2.15) along with boundary conditions (3.10) gives

$$
\frac{\partial}{\partial r}\left(\frac{1}{r} \frac{\partial \psi}{\partial r}\right)-\frac{x^{2}}{r} \psi=\frac{r}{2} c_{1}
$$

where $c_{1}$ is an arbitrary function of $z$. Equation (4.1) after using the transformation

$$
\phi_{1}=\frac{\psi}{r}+\frac{r}{2 \chi^{2}} c_{1}
$$

can be reduced into the following modified Bessel equation:

$$
r^{2} \frac{\partial^{2} \phi_{1}}{\partial r^{2}}+r \frac{\partial \phi_{1}}{\partial r}-\left(x^{2} r^{2}+1\right) \phi_{1}=0
$$

whose solution along with (4.2) and boundary conditions (3.10) is given below:

$$
\psi=\frac{r\left[r F h I_{0}(X h)+h r I_{1}(X h)-\left(2 F+h^{2}\right) I_{1}(x r)\right]}{h^{2} \chi I_{2}(X h)},
$$

where $I_{0}, I_{1}$, and $I_{2}$ are the modified Bessel function of order zero, one, and two, respectively.

Substitution of (4.4) into (2.8) and (2.13) yields the following expressions for axial velocity $(w)$ and axial pressure gradient:

$$
\begin{gathered}
w=\frac{2 F X I_{0}(\chi h)+2 h \chi I_{1}(X h)-2 \chi\left(2 f+h^{2}\right) I_{0}(\chi r)}{h^{2} \chi I_{2}(\chi h)}, \\
\frac{d p}{d z}=\frac{-\chi^{2}\left(2 F+h^{2}\right) I_{0}(\chi r)}{h^{2} I_{2}(x h)}
\end{gathered}
$$




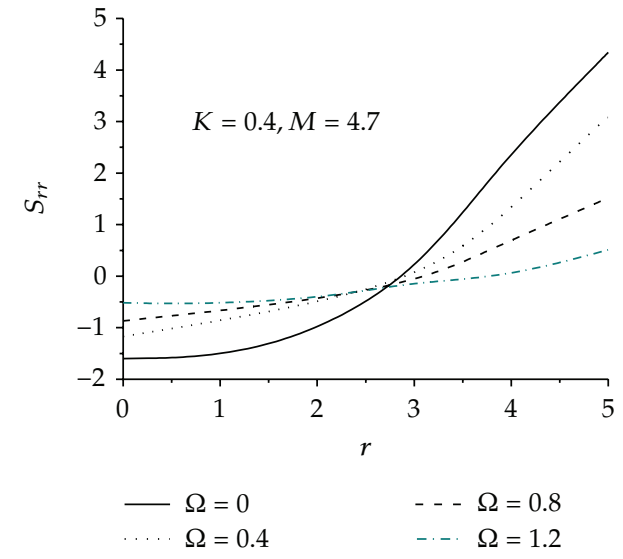

(a)

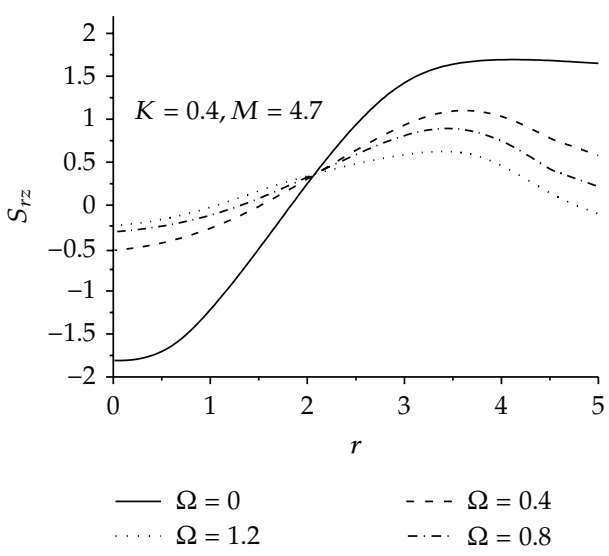

(b)

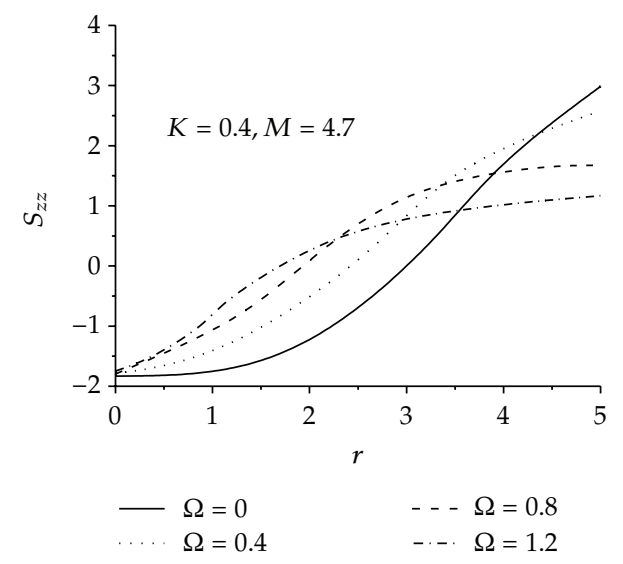

(c)

Figure 2: Show the stress distributions for tube $a=0.3, b=0.4$, and $d=1.1$.

The expressions for pressure rise $\left(\Delta P_{\lambda}\right)$ and frictional force $\left(F_{\lambda}\right)$ per wavelength are, respectively, given by

$$
\begin{gathered}
\Delta p_{\curlywedge}=\int_{0}^{2} \frac{d p}{d z} d z \\
F_{\curlywedge}=\int_{0}^{2 \pi} h^{2}\left(-\frac{d p}{d z}\right) d z
\end{gathered}
$$

\section{Results and Discussion}

To investigate the effects of rotation $(\Omega)$, magnetic parameter $(M)$, material parameter $\left(\lambda_{1}\right)$, permeability of the porous medium $(k)$, and mean flux $(F)$, we plotted Figures 2-6.

The stress distribution $\left(S_{r r}\right),\left(S_{r z}\right)$, and $\left(S_{z z}\right)$ in tube for different values of the rotation $(\Omega)$ is presented in Figures 2(a), 2(b), and 2(c), respectively. We notice that the stress is 

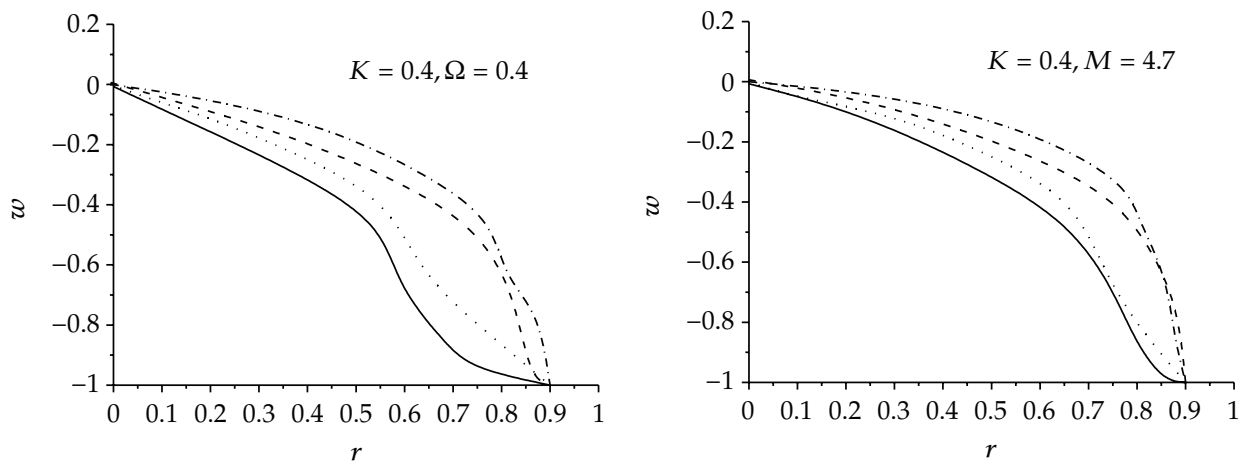

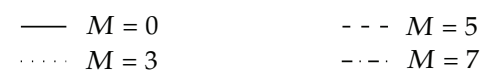

(a)

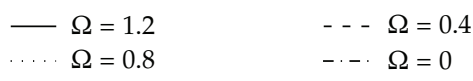

(b)

Figure 3: Show the velocity distributions for $a=0.3, b=0.4, d=1.1$, and $\lambda=0.5$.
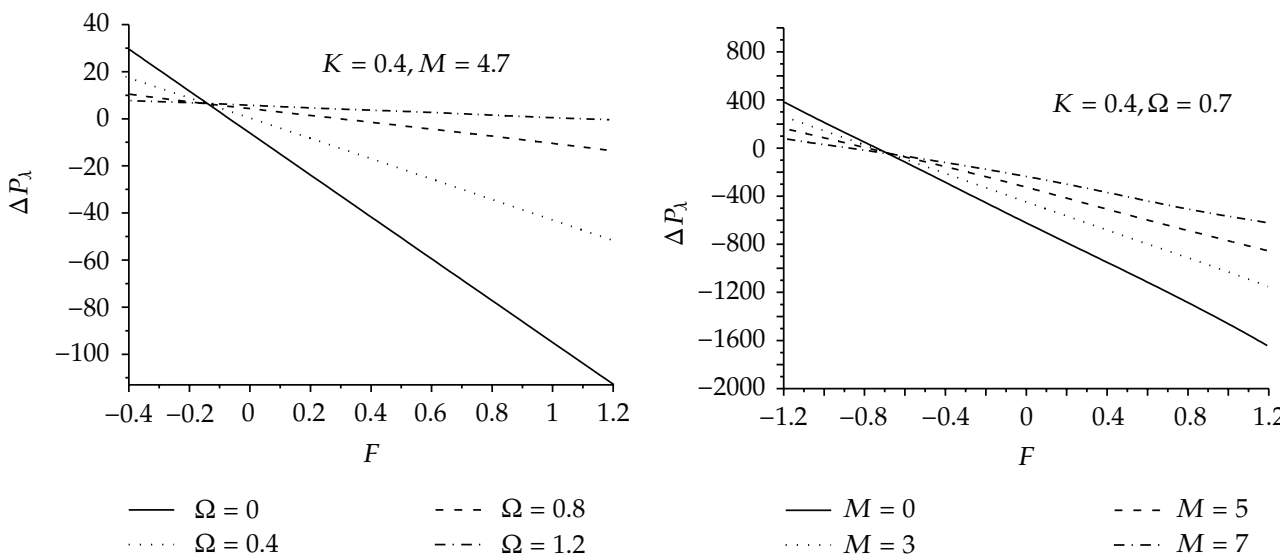

(a)

(b)

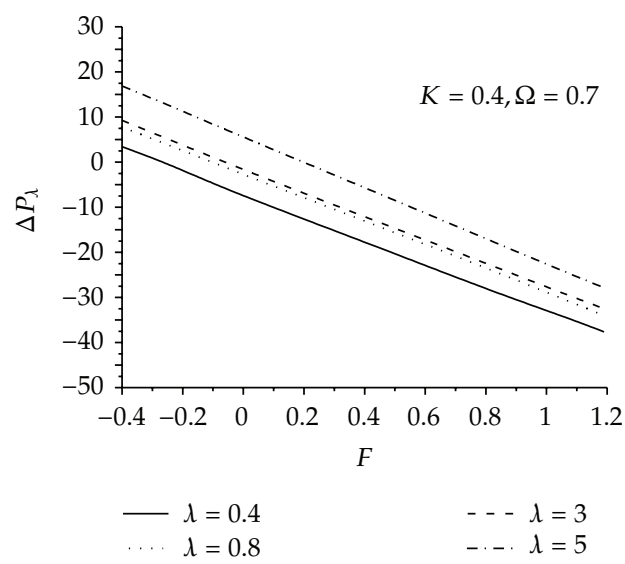

(c)

Figure 4: Show the Graph of pressure rise versus $F$ in symmetric channels for $a=0.3, b=0.4$, and $d=1.1$. 


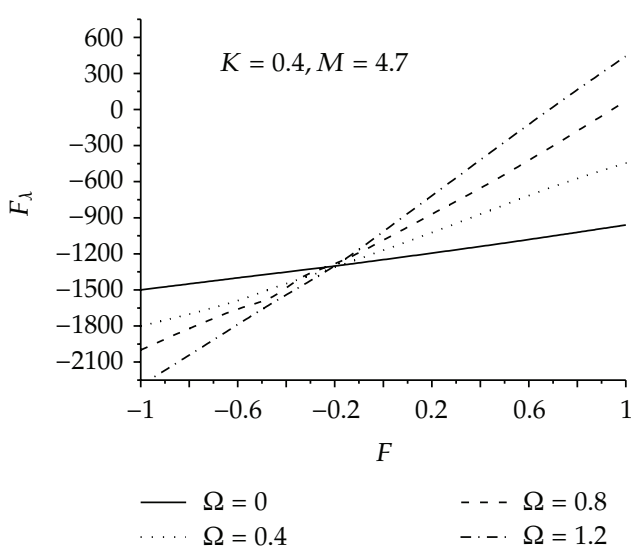

(a)

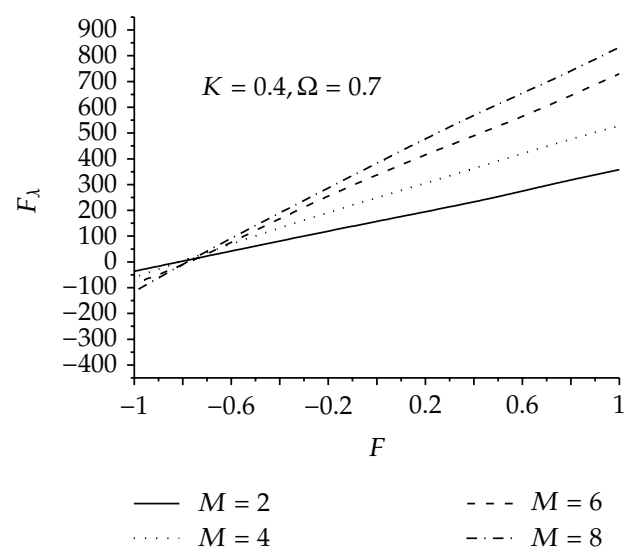

(b)

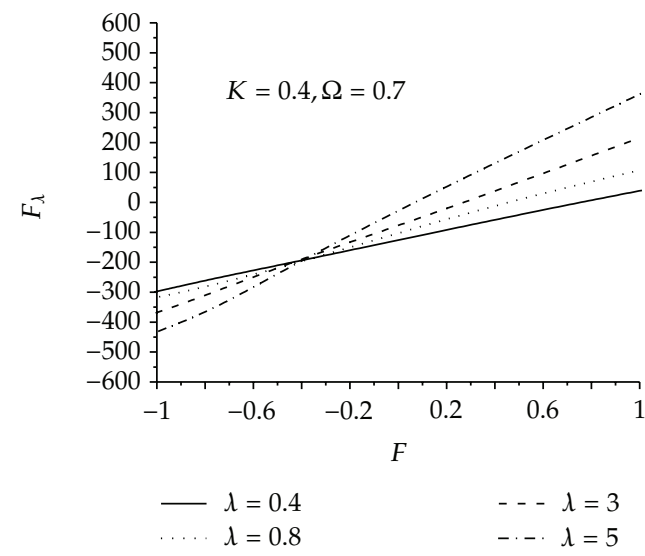

(c)

Figure 5: Plot showing $F_{\lambda}$ for $F \in[1,1]$ for changing rotation $(\Omega)$, (a), Hartman number $M(b)$, and material parameter $\lambda_{1}$ (c).

in oscillatory behaviour, which may be due to peristalsis. The absolute value of stress distribution $\left(S_{r r}\right),\left(S_{r z}\right)$, and $\left(S_{z z}\right)$ increases at first with increasing the rotation $(\Omega)$, and then it decreases with increasing the rotation $(\Omega)$ when large values of $r$ have been taken into account. It is observed that the absolute values of the stress are larger in case of a Jeffrey fluid when compared with Newtonian fluid.

The effects of the rotation $(\Omega)$ and magnetic parameter $(M)$ on the velocity is plotted in Figure 3. Figure 3 shows that influence of the rotation $(\Omega)$ and magnetic parameter $(M)$ on the velocity increases with the increase of magnetic parameter $(M)$, and it decreases with the increase of the rotation $(\Omega)$.

Figure 4 shows the variation of $\Delta P_{\lambda}$ with flow rate $F$ for values of rotation $(\Omega)$, magnetic parameter $(M)$, and material parameter $\left(\lambda_{1}\right)$ for tube. We observe that the peristaltic pumping rate increases with increase of magnetic parameter $(M)$ and material parameter $\left(\lambda_{1}\right)$, and it decreases with the increase of the rotation $(\Omega)$. The phenomenon of trapping is another interesting topic in peristaltic transport. The formation of an internally circulating bolus of the fluid by closed streamlines is called trapping, and this trapped bolus pushed ahead along the peristaltic wave. 


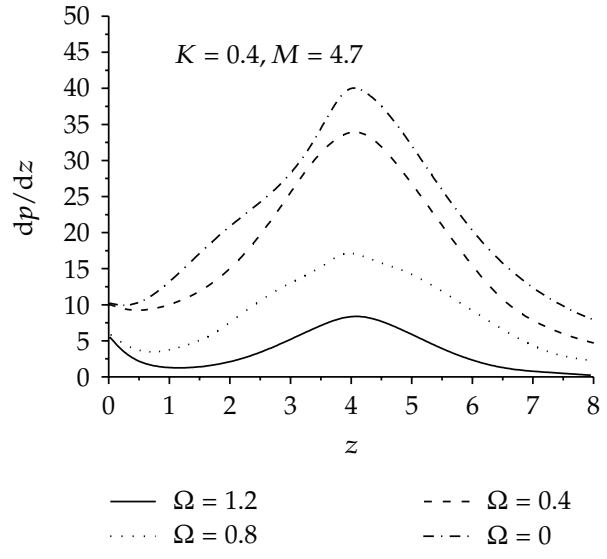

(a)

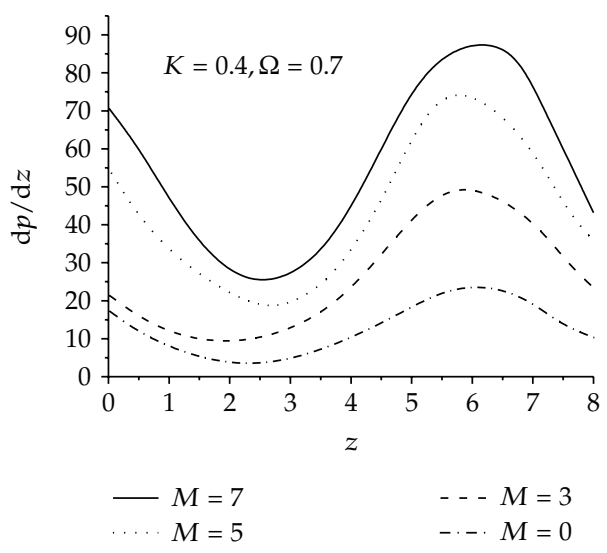

(b)

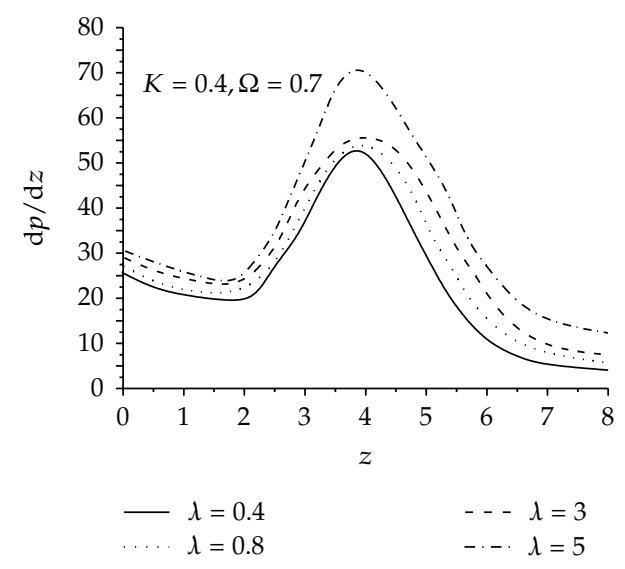

(c)

Figure 6: Plot showing variation of the pressure gradient $(\mathrm{d} p / \mathrm{d} z)$ within wavelength for various values of rotation $(\Omega)\left(\right.$ a) magnetic parameter $(M)(b)$, and material parameter $\left(\lambda_{1}\right)(\mathrm{c})$.

Figure 5 shows the variation of $F_{\lambda}$ with flow rate $F$ for values of rotation $(\Omega)$, magnetic parameter $(M)$, and material parameter $\left(\lambda_{1}\right)$ for tube. Figures $5(\mathrm{a}), 5(\mathrm{~b})$, and $5(\mathrm{c})$ display the influence of $(\Omega)$, magnetic parameter $(M)$, and material parameter $\left(\lambda_{1}\right)$, respectively, for tube on $F_{\lambda}$. Figure 5(a) refers to the case when $F=-0.2$. Here it is noted that $F_{\lambda}$ increases with decrease of rotation $(\Omega)$ when $-0.6 \leq F \leq-0.2$ and it increases with the increase of the rotation $(\Omega)$ when $-0.2<F$. Figure $5(\mathrm{a})$ refers to the case when $\Omega=1.2$, here, it is noted that $F_{\lambda}$ is negative and positive when $-0.6 \leq F \leq 0.6$ and $0.6<F$, respectively. When $\Omega=0.8$. Here it is noted that $F_{\lambda}$ is negative and positive when $-0.6 \leq F \leq 0.83$ and $0.83<F$, respectively. When $\Omega=0.4, F_{\lambda}$ is negative for $-0.6 \leq F \leq 1.4$ and positive for $1.4<\mathrm{F}$. Also, when $\Omega=0.0$, $F_{\mathcal{\lambda}}$ is negative for $-0.6 \leq F \leq 3.0$ and positive for $3.0<F$. Figure $5(\mathrm{~b})$ refers to the case when $F=-0.8$. Here it is noted that $F_{\lambda}$ increases with decrease of magnetic parameter $(M)$ when $-1.5 \leq F \leq-0.8$, and it increases with the increase of the magnetic parameter $(M)$ when $-0.8<F$. Figure $5(\mathrm{c})$ refers to the case when $F=-0.8$. Here it is noted that $F_{\mathcal{\lambda}}$ increases with decrease of material parameter $\left(\lambda_{1}\right)$ when $-1 \leq F \leq-0.48$, and it increases with the increase of the magnetic parameter $(M)$ when $-0.48<F$. 
Figure 6 shows the distributions of the pressure gradient within a wavelength for various values of the rotation $(\Omega)$, magnetic parameter $(M)$ and material parameter $\left(\lambda_{1}\right)$. The effects of magnetic parameter $(M)$, on the pressure gradient $(\mathrm{d} p / \mathrm{d} z)$ within a wavelength are plotted in Figure 2(b). It is noticed that magnetic parameter $(M)$ and material parameter $\left(\lambda_{1}\right)$ increase the maximum amplitude of $(\mathrm{d} p / \mathrm{d} z)$ when compared to the case with zero magnetic parameter and zero material parameter $\left(\lambda_{1}\right)$.

\section{Conclusion}

The influence of the rotation $(\Omega)$, magnetic parameter $(M)$, and material parameter $\left(\lambda_{1}\right)$ on the peristaltic flow of a Jeffrey fluid in tube has been analyzed. The analytical expressions are constructed for axial velocity, $F_{\lambda}$, and pressure gradient. Numerical investigation is plotted and discussed. The main findings can be summarized as follows.

(i) The axial velocity for the MHD fluid is less when compared with hydrodynamic fluid in the central part of the tube.

(ii) The magnitude of $(\mathrm{d} p / \mathrm{d} z)$ and $F_{\lambda}$ increases with increase of magnetic parameter $(M)$ and material parameter $\left(\lambda_{1}\right)$ and it increases with decrease of the rotation $(\Omega)$.

(iii) The size of trapped bolus is smaller in Jeffrey fluid when compared with that of Newtonian fluid $\left(\lambda_{1}=0\right)$.

(iv) The magnitudes of $(\mathrm{d} p / \mathrm{d} z), \Delta P_{\lambda}$, and $F_{\lambda}$ for Newtonian fluid are smaller than that of Jeffrey fluid.

(v) For large values of magnetic parameter $(M)$ and material parameter $\left(\lambda_{1}\right)$, the magnitudes of $\Delta P_{\lambda}$ and $\Delta P_{\lambda}$ increase with decrease of the rotation $(\Omega)$.

\section{References}

[1] S. R. Mahmoud, A. M. Abd-Alla, and N. A. AL-Shehri, "Effect of the rotation on the radial vibrations in a non-homogeneous orthotropic hollow cylinder," International Journal of Modern Physics B. In press.

[2] S. R. Mahmoud, A. M. Abd-Alla, and B. R. Matooka, "Effect of the rotation on wave motion through cylindrical bore in a micropolar porous cubic crystal," International Journal of Modern Physics B, vol. 25, no. 20, pp. 2713-2728, 2011.

[3] S. R. Mahmoud, A. M. Abd-Alla, and N. A. AL-Shehri, "Effect of rotation on thermoelastic waves in a non-homogeneous infinite cylinder," The Open Mechanics Journal, vol. 4, pp. 58-64, 2010.

[4] A. M. Abd-Alla, S. R. Mahmoud, and N. A. AL-Shehri, "Effect of the rotation on a non-homogeneous infinite cylinder of orthotropic material," Applied Mathematics and Computation, vol. 217, no. 22, pp. 8914-8922, 2011.

[5] A. M. Abd-Alla, S. R. Mahmoud, and M. I. R. Helmy, "Influences of rotation, magnetic field, initial stress, and gravity on Rayleigh waves in a homogeneous orthotropic elastic half-space," Applied Mathematical Sciences, vol. 4, no. 1-4, pp. 91-108, 2010.

[6] A. M. Abd-Alla and S. R. Mahmoud, "Magneto-thermoelastic problem in rotating non-homogeneous orthotropic hollow cylinder under the hyperbolic heat conduction model," Meccanica, vol. 45, no. 4, pp. 451-462, 2010.

[7] A. M. Abd-Alla and S. R. Mahmoud, "Effect of the rotation on propagation of thermoelastic waves in a non-homogeneous infinite cylinder of isotropic material," International Journal of Mathematical Analysis, vol. 4, no. 42, pp. 2051-2064, 2010.

[8] S. R. Mahmoud, "Effect of rotation on generalized magneto-thermoelastic rayleigh waves in a granular medium under influence of gravity field and initial stress," Applied Mathematical Sciences, vol. 5, no. 41, pp. 2013-2032, 2011. 
[9] N. A. Afifi, S. R. Mahmoud, and H. M. Al-Isede, "Effect of magnetic field and wall properties on peristaltic motion of micropolar fluid in circular cylindrical tubes," International Mathematical Forum, vol. 6, no. 27, pp. 1345-1356, 2011.

[10] N. A. S. Afifi and N. S. Gad, "Interaction of peristaltic flow with pulsatile magneto-fluid through a porous medium," Acta Mechanica, vol. 149, no. 1-4, pp. 229-237, 2001.

[11] N. A. S. Afifi, "Aspects of a magneto-fluid with suspended particles," Applied Mathematics $\mathcal{E}$ Information Sciences, vol. 1, no. 1, pp. 103-112, 2007.

[12] T. Hayat, M. Khan, and M. Ayub, "Couette and Poiseuille flows of an Oldroyd 6-constant fluid with magnetic field," Journal of Mathematical Analysis and Applications, vol. 298, no. 1, pp. 225-244, 2004.

[13] C. Fetecau and C. Fetecau, "On some axial Couette flows of non-Newtonian fluids," Zeitschrift für Angewandte Mathematik und Physik (ZAMP), vol. 56, no. 6, pp. 1098-1106, 2005.

[14] W. C. Tan and T. Masuoka, "Stokes first problem for second grade fluid in a porous half space," International Journal of Non-Linear Mechanics, vol. 40, no. 4, pp. 515-522, 2005.

[15] A. M. Siddiqui and W. H. Schwarz, "Peristaltic motion of a third-order fluid in a planar channel," Rheologica Acta, vol. 32, no. 1, pp. 47-56, 1993.

[16] A. M. Siddiqui and W. H. Schwarz, "Peristaltic flow of a second-order fluid in tubes," Journal of NonNewtonian Fluid Mechanics, vol. 53, pp. 257-284, 1994.

[17] R. A. Ramachandra and S. Usha, "Peristaltic transport of two immiscible viscous fluids in a circular tube," Journal of Fluid Mechanics, vol. 298, pp. 271-285, 1995.

[18] T. Hayat, Y. Wang, K. Hutter, S. Asghar, and A. M. Siddiqui, "Peristaltic transport of an Oldroyd-B fluid in a planar channel," Mathematical Problems in Engineering, no. 4, pp. 347-376, 2004.

[19] A. M. Siddiqui and W. H. Schwarz, "Peristaltic flow of a second-order fluid in tubes," Journal of NonNewtonian Fluid Mechanics, vol. 53, pp. 257-284, 1994.

[20] Kh. S. Mekheimer, "Peristaltic flow of blood under effect of a magnetic field in a non-uniform channels," Applied Mathematics and Computation, vol. 153, no. 3, pp. 763-777, 2004.

[21] Kh. S. Mekheimer, "Peristaltic transport of a couple stress fluid in a uniform and non-uniform channels," Biorheology, vol. 39, no. 6, pp. 755-765, 2002.

[22] T. Hayat and N. Ali, "Peristaltic motion of a Jeffrey fluid under the effect of a magnetic field in a tube," Communications in Nonlinear Science and Numerical Simulation, vol. 13, no. 7, pp. 1343-1352, 2008.

[23] T. Hayat, Y. Wang, A. M. Siddiqui, K. Hutter, and S. Asghar, "Peristaltic transport of a third-order fluid in a circular cylindrical tube," Mathematical Models E Methods in Applied Sciences, vol. 12, no. 12, pp. 1691-1706, 2002.

[24] A. M. Siddiqui, T. Hayat, and M. Khan, "Magnetic fluid model induced by peristaltic waves," Journal of the Physical Society of Japan, vol. 73, pp. 2142-2147, 2004.

[25] K. H. S. Mekheimer, "Non-linear peristaltic transport of magneto-hydrodynamic flow in aninclined planar channel," Arabian Journal for Science and Engineering, vol. 28, no. 2A, pp. 183-201, 2003. 


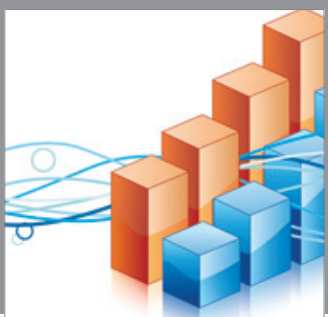

Advances in

Operations Research

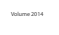

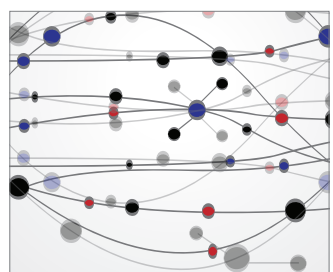

\section{The Scientific} World Journal
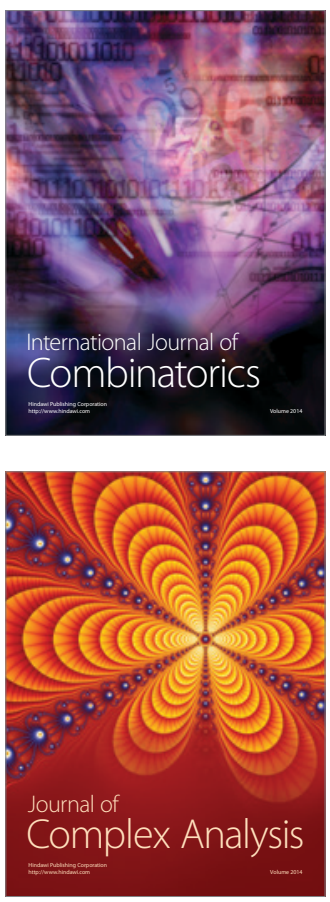

International Journal of

Mathematics and

Mathematical

Sciences
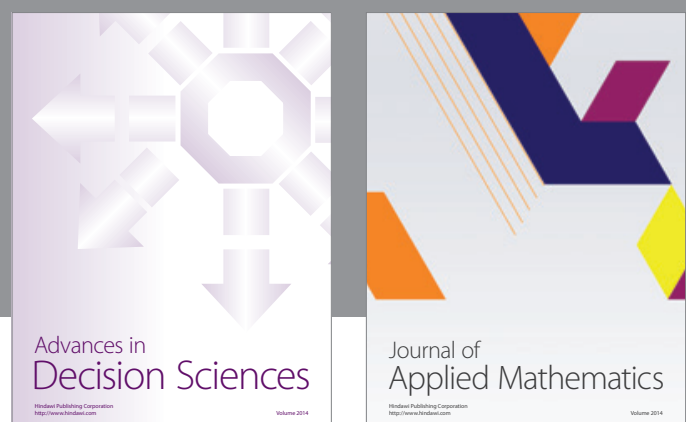

Journal of

Applied Mathematics
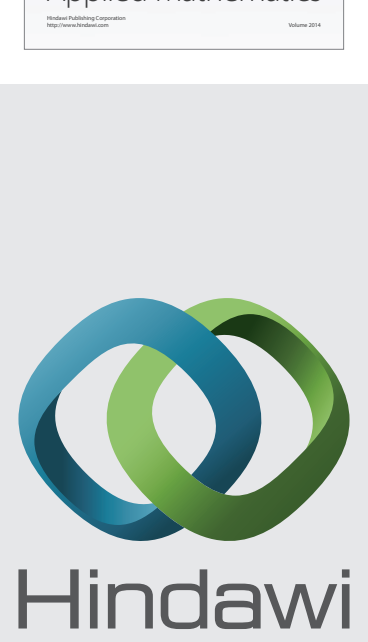

Submit your manuscripts at http://www.hindawi.com
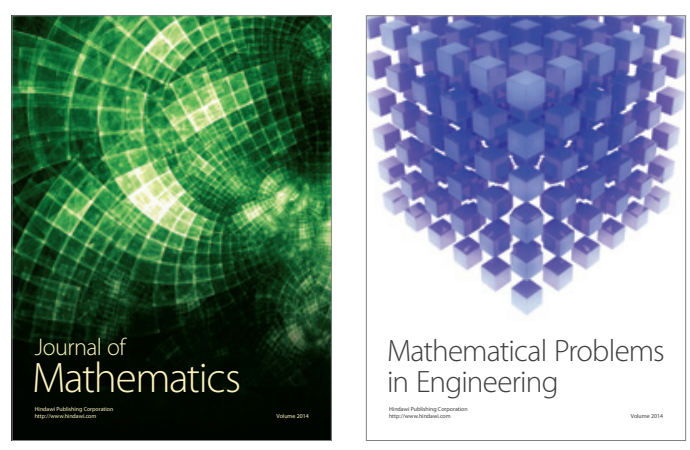

Mathematical Problems in Engineering
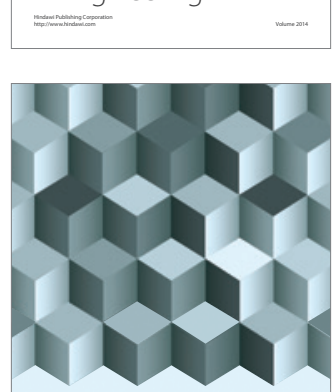

Journal of

Function Spaces
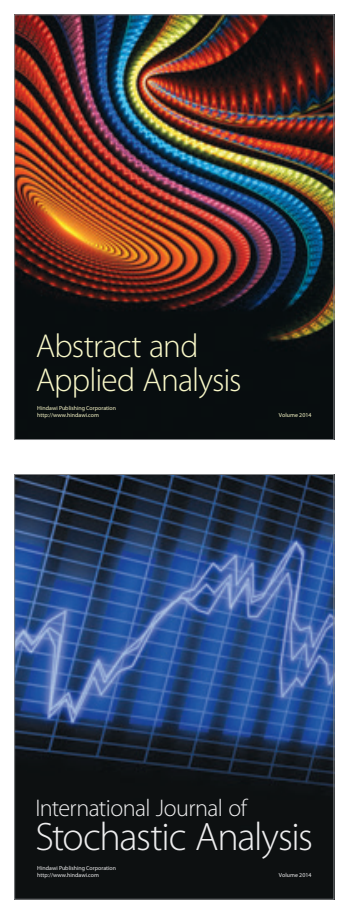

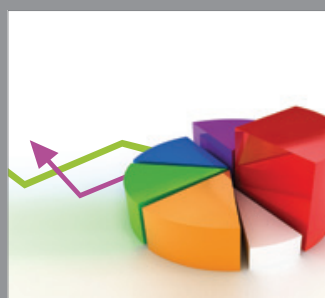

ournal of

Probability and Statistics

Promensencen
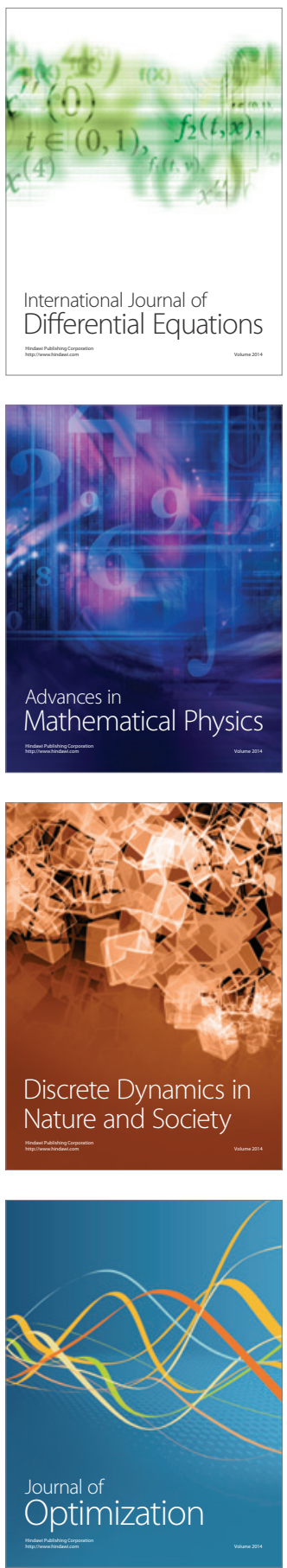